\title{
Scattered Kaon Study in the KOTO Experiment at J-PARC
}

\section{Stephanie Su $\mathbf{u}^{* \dagger}$}

Department of Physics, University of Michigan

E-mail: stephsulumich.edu

The KOTO experiment at J-PARC aims to explore physics beyond the Standard Model (SM) by measuring the branching ratio of the $K_{L} \rightarrow \pi^{0} v \bar{v}$ decay, predicted by the SM to be $(3.0 \pm 0.3) \times 10^{-11}[1]$ and is characterized by a pair of photons from the $\pi^{0}$ decay and no other detected particles. The decay is identified by observing two photons with a large transverse momentum on an undoped Cesium Iodide (CsI) electromagnetic calorimeter and no other signals on the hermetic veto counters around the decay region.

Kaons which decay outside the beam line with two photons in the final state, such as $K_{L} \rightarrow \gamma \gamma$ and $K_{L} \rightarrow \pi^{+} \pi^{-} \pi^{0}$, can appear to have a large transverse momentum due to kaon scattering and beam interaction with the detector components. These off-axis kaon decay events can impact the measurement of the $K_{L} \rightarrow \pi^{0} v \bar{v}$ branching ratio. An Aluminum target inserted at the upstream of the KOTO detector was used to study the kaon beam profile, which provided the vertex information of off-axis kaon decays. The beam profile provided insights on background contributions to the signal. Studies on the kaon beam profile and background estimation from kaon scattering is presented in this paper.

ICHEP 2018, 39th International Conference on High Energy Physics

4-11 July 2018

Seoul, Korea

\footnotetext{
${ }^{*}$ Speaker.

${ }^{\dagger}$ for the KOTO collaboration.
} 


\section{Introduction}

In the KOTO experiment, 3 the $K_{L} \rightarrow \pi^{0} v \bar{v}$ signal is iden-

\section{sumes the decay originated from} the neutral beam line. Conse-

\section{Methods} were applied in this study.

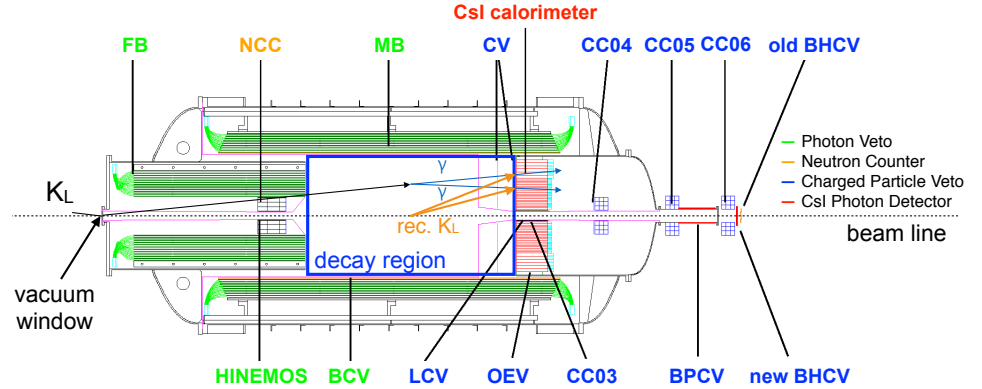

Figure 1: Illustration of a scattered $K_{L} \rightarrow \gamma \gamma$ being reconstructed onto the beam line. quently, decay modes with two photons in the final state and decay off the beam line, such as scattered $K_{L} \rightarrow \gamma \gamma$ and $K_{L} \rightarrow \pi^{+} \pi^{-} \pi^{0}$ decays from scattered kaons, can appear to possess a large transverse momentum in the event reconstruction, as shown in Fig. 1.

A removable Aluminum target (Al-target) located in front of the vacuum window was used to study the beam profile of scattered kaons. Backgrounds from the scattered kaons at the vacuum window can be estimated and scaled using the material density and thickness between the Al-target and the vacuum window, which was made of Kapton. The $K_{L} \rightarrow 3 \pi^{0}$ events were selected for this study because the background contamination was small. Instead of using the beam line constraint, this study reconstructed events onto the Center-of-Energy (COE) axis, where the COE position was defined as the weighted average of electromagnetic shower positions on the CsI with their energies. The reconstructed $K_{L}$-decay vertex positions and momentum were fed into Monte Carlo simulations as seeds to generate scattered kaons. Several event selection criteria on $K_{L} \rightarrow \pi^{0} v \bar{v}$

\section{Results}

The $K_{L}$ scattering rate at the vacuum window, calculated and scaled from the Al-target using $K_{L} \rightarrow 3 \pi^{0}$ events, was $4.66 \times 10^{-4}$. In the 2015 runs, corresponding to $4.58 \times 10^{12}$ kaons with the single event sensitivity of $1.30 \times 10^{-9}$, the scattered $K_{L} \rightarrow \gamma \gamma$ background contribution was $0.014 \pm 0.001$, as shown in Fig. 2. Veto detectors effectively reduced scattered $K_{L} \rightarrow$ $\pi^{+} \pi^{-} \pi^{0}$ background, and the upper limit from this background was calculated to be $7 \times 10^{-13}$.

\section{References}

[1] A. J. Buras, D. Buttazzo, and R. Knegjens, J. High Energ. Phys. 2015, 166 (2015).

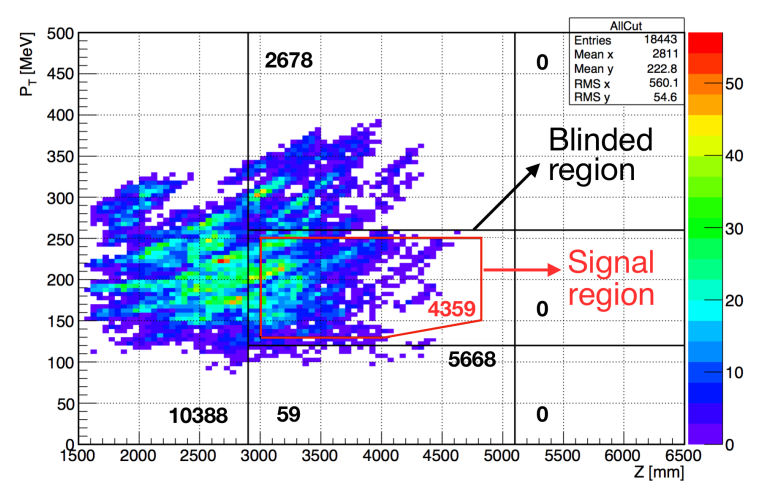

Figure 2: Remaining $K_{L} \rightarrow \gamma \gamma$ background distribution from $6 \times 10^{7}$ scattered kaons on the reconstructed $P_{T}-Z$ plane. The number of events in each region was shown. 